Schtschepotjew: Selbstständige Contraction der Herzspitze etc. $\mathbf{5 3}$

$$
\begin{array}{cc}
\text { 7,5 gr A.-R. } & 7,5 \text { dsgl. } \\
\text { mit Kalkwasser: } & \text { mit Barytwasser: } \\
2,316 \mathrm{gr} & 2,274 \mathrm{gr} \\
=30,9 \text { Pre. } & =30,3 \mathrm{Prc} .
\end{array}
$$

gefällte Proteinsubstanz, während Kaliwasser 32,3 Prc. ergeben hatte. In der Fällungsflüssigheit blieben bedeutende Mengen Proteinsubstanz gelöst.

Die näheren Untersuchungen können in einiger Zeit erst beendet werden.

\title{
Selbstständige Contraction der Herzspitze, Veränderungen der Muskeln und der weissen Blut- körperchen unter dem Einfluss von Chinin.
}

$$
\text { Von }
$$

Nic. Schtschepotjew.

Zahlreiche, in der medicinischen Litteratur vorhandene, die Wirkung des Chinins auf den thierischen Organismus behandelnde Untersuchungen haben zu folgenden Resultaten geführt: 1) Die Wirkung des Chining auf weisse Blutkörperchen äussert sich in Hemmung oder gänzlicher Aufhebung ihres Vermögens amöboide Bewegungen anszuführen; 2) es wirkt stark auf niedere Organismen ein, verlangsamt oder 'hindert Gährung und Fäulniss; 3) es setzt die Körpertemperatur herab; 4) Herzcontractionen werden von ihm beschleunigt oder verlangsamt (je nach der Dosis); 5) es setzt die Reizbarkeit der Herzmuskelfaser stark herab (Joly et, Le vizki, A, Eụlenburg) ohne eine Einwirikung auf die Skelettmuskeln zu 
zeigen (Levizki), oder letztere werden eher als die Herzmuskelfasern in ihrer Function abgeändert (Eulenburg); 6) nach einigen Forschern wird der Blutdruck durch Chinin herabgesetzt (Levizki, Schroff), nach andern ernöht (Bordier); 7) das Chinin wirkt auch auf das Nervensystem (Cha peron, Eul enburg); 8) endlich ruft es Milzcontraction hervor (Kü chenmeister, Botkin u. and.).

Ohne den unzweifelhaften Werth obiger Untersuchungen zu bestreiten, muss doch auf die Verschiedenheit der Resultate und darauf, dass sie uns über die nächste. Ursache der Chininwirkung auf den thierischen Organismus im Ungewissen lassen, hingewiesen werden.

Meine, auf Vorschlag des Herrn Prof. Dogiel, in dessen Laboratorium vorgenommenen, dieselbe Frage betreffenden Untersuchungen werden jedenfalls nicht ohne Nutzen für weitere Forschungen dieses zar Heilung verschiedener Krankheiten unumgänglich nöthigen Mittels bleiben. Diese Untersuchungen behandeln nur die Wirkung des Chinins auf weisse Blutkörperchen, Herz und Muskeln.

a) Wirkung des Chinins auf weisse Blutkörperchen.

Meine Untersuchungen iuber dio Wirkung des Chinins auf weisse Blutkörperchen bestanden in mikroskopischen Beobachtungen (feuchte Kammer) des Blutes von Fröschen, welche mit schwefeloder salzsaurem Chinin von neutraler Reaction, hypodermatisch, vergiftet waren.

Das Blut zur Beobachtung wurde mittelst eines Glascapillarröhrchens direct aus dem Herzen der mit Chinin vergifteten Frösche, indem man den Ventrikel durchstiess, genommen. Meine in dieser Art angestellten Versuche führten zum folgenden Resultat. Kleine, die Circulation wenig beeinflussende $(0,001-0,005 \mathrm{gr})$ und mittlere $(0,006-0,014 \mathrm{gr})$, die Herzcontractionen deutlich verlangsamende, ebenfalls die Reflexe herabsetzende Dosen Chinins zeigen keinen bemerkenswerthen Einfluss auf die weissen Blutkörperchen.

Obwohl sie in diesem Falle mehr gekörnt, und gerundeter erscheinen, bleibt ihre Fähigkeit zu amöboiden Bewegungen doch in voller Kraft. Bei Vergiftung der Frösche mit grossen Dosen 
Chinins $(0,018-0,025 \mathrm{gr})$, welche nach Verlauf von $1^{1 / 2}-2$ Stunden auffallende Verlangsamung ind theilweise oder gänzliche Aufhebung der Reflexe herbeiführen, sieht man, dass die amöboiden Bewegungen der weissen Blutkörperchen nicht nur geschwächt sind, sondern zeitweilig ganz sistiren. 3-5 Minuten liegen sie wie erstarrt; darnach erscheinen wieder Bewegungen, welche allmählig dentlicher werden. Auf Grund ähnlicher Beobachtungen kann man unmöglich mit dem Schluss, dass kleine und mittlere Dosen Chinins, hypodermatisch den Fröschen applicirt, schon stark auf die weissen Blutkörperchen einwirke, ihre Fähigkeit zu amöhoiden Bewegungen aufhebe und ihre Emigration aus den Gefässen verhindere, übereinstimmen. Wird überhaupt die Emigration der weissen Blutkörperchen aus den Gefässen aufgehalten, so nimmt hieran wahrscheinlich nicht wenig eine Veränderung der Gefässwandungen selbst, im Sinne von Ap pert ${ }^{1}$ ) und Jahn ${ }^{2}$ ), Theil.

In jedem Falle ist die Erklärung einiger Functionsveränderungen des thierischen Organismus allein durch die Wirkung des Chinins auf weisse Blutkörperchen nicht vollkommen gerechtfertigt. Wenn auch Chinin anf das Blut eines lebenden Thieres so stark einwirken würde, dass man dadurch das Fallen der Temperatur, die Abnahme der Eiterungen, etc. erklären könnte, so müsste man doch ähnliche Veränderungen des Blutes eher an seinen übrigen Bestandtheilen, als ausschliesslich an den weissen Körperchen suchen. Die Veränderungen in der Grösse der rothen Blutkörperchen, wie es Manas sein ${ }^{3}$ ) angiebt, sind auch zweifelhaft, wenigstens ist nach Beobachtung von Prof. Dogiel ihre Messung so schwierig, dass man mit grosser Vorsicht die auf solche Weise erhaltenen Resultate über die Grössenverhältnisse der rothen Blutkörperchen aufnehmen muss. Mehr Anspruch auf Wahrscheinlichkeit haben die Beschreibungen von Schmiedeberg ${ }^{4}$ ), Bonwets $\mathrm{ch}^{5}$ ) und $B$ inz ${ }^{6}$ ), welche dem Chinin eine Beziehung zum Sauerstoff

1) Virchow's Archiv. 1877.

2) Berliner Klinik W. S. 1872.

3) Ueber die Dimensionen der rothen Blutkörperchen unter verschiedenen Verhältnissen. Berlin 1872.

4) Petersburg. med. Zeitschr. 1868. Bd. XIV.

5) Ueber den Einfluss versch. Stoffe auf die Umsetzung des Sauerstoffs im Blute. Diss. 1869 .

6) Ueber Chinin und Blut. Arch. f. Exp. Path. u. Pharm. Bd. I. 1873 
des Hämoglobins vindiciren. Wenn die weissen Blutkörperchen auch durch grosse Dosen Chinins ihre Fähigkeit zu amöboiden Bewegungen verlieren, so tritt ja auch diese Erscheinung unter anderen Umständen ein: nach Einwirkung niederer Temperaturgrade, der Blausäure, des Aethylalkohols und wahrscheinlich noch vieler anderer Körper. Mit Sicherheit kann ich es von Blausäure und Aethylalkohol behaupten. Wenn man einen Frosch in verdiinnten Aethylalkohol (30\% nach Tralles) legt und den Eintritt starker oder völliger Anaesthesie abwartet, darauf ihn herausnimmt und abwäscht, so erhält man bei mikroskopischer Betrachtung des aus dem Herzen genommenen Blutes folgendes Bild. Die weissen Blutkörperchen sind stark gekörnt; 2-4 Minuten liegen sie wie erstarrt, worauf wieder Fortsätze ausgeschickt werden und andere, mannigfache Formveränderungen auftreten. Aehnlich dem Aethyla]kohol wirkt auf die weissen Blutkörperchen die Blausäure, wovon man sich bei mikroskopischer Untersuchung des direct aus dem Herzen genommenen Blutes von Fröschen, welche durch hypodermatische Anwendung des Kirschlorbeerwassers vergiftet sind, nachdem das Reflexvermögen schon geschwächt ist, ibberzeugen kann.

b) Veränderungender Herzcontractionendureh $\mathrm{Ch}$ in in.

Die Beobachtungen von Schlocko ${ }^{1}$ ), A. Eulenburg ${ }^{2}$ ), Mendenhal13) u. A. ergaben, dass kleine Dosen Chinins den Puls beschleunigen, grosse dagegen verlangsamen. Meine an Fröschen, Kaninchen, Hunden und mir selbst angestellten Versuche erlauben die Schlussfolgerung im Allgemeinen, dass die Wirkung des Chinins auf das Herz in Aenderung der Zahl und Kraft der Contractionen besteht, obwohl die Wirkung auf's Froschherz von der auf das Herz der Hunde und des Menschen etwas sich unterscheidet. Kleine Chinindosen rufen selten bei Fröschen eine Beschleunigung der Herzcontractionen hervor. Grösstentheils tritt bei diesen Thieren ebenso von kleinen und mittleren, wie von

1) Studien d. physiol. Inst. zu Breslau. Heft I. S. 163.

2) Reichert's u. du Bois-Reymond's Arch. 1865. S. 426.

3) Americ. Journal. Juli 1846. 
grossen Gaben Chinin eine Verlangsamung der Herzschläge ohne vorhergehende Beschleunigung auf. 14 über die Wirkung des Chinins auf Herzcontractionen an Hunden angestellte Versuche führten mich zu der Ueberzeugung, dass dieselben sowohl von grossen wie von kleinen Dosen beschleunigt werden. Zum Beweis will ich hier einige Versuche an Fröschen, Hunden und mir selbst vorführen.

Versuche an Fröschen. Versuch I. Frosch mittlerer Grösse, an ein Korktäfelchen in der Rückenlage befestigt, das Herz freigelegt; nachdem ihm Zeit zur Erholung von der Operation gegönnt, wurde die Zahl der Herzschläge mittelst eines Chronometers bestimmt. Zahl der Herzschläge' in 30 Secunden $=22$. Nach subcutaner Application von 0,002 chinini muriatici am Schenkel, wurden die Herzcontractionen folgender Art bestimmt.

Beobachtungszeit in Minuten nach Chininapplication.

$$
7
$$

14

30
Zahl der Herzcontractionen in 30 Secunden.

Versuch II. Kleiner Frosch; das Herz ohne Verletzung des Pericardium freigelegt; Erholung und Bestimmung der Herzschläge $=21$ in 30". Nach subcutaner Application von 0,003 chin. muriat:

Beobachtungszeit in Minuten nach

Chininapplication.

$$
8
$$

20

36

50
Zahl der Herzcontractionen in 30 Secunden.

Versuch III. Frosch mittlerer Grösse. Zahl der Herzschläge nach Eröffnung dex Brusthöhle und Erholung in 30" $=19$. Nach subcutaner Application von chin. muriat. am Schenkel, waren die Herzschläge:

Beobachtungszeit in Minuten.

10

20

40

55
Zahl der Herzcontractionen in 30".

17

15

14

14

Versuch IV. Bei einem mittelgrossen Frosch das Herz mit Entfernung des Pericardium freigelegt. Erholung von der Operation. Zahl der Herzschläge $=20$ in 30". Nach subcutaner Application von 0,020 chin. muriat. Beobachtungszeit in Minuten nach Zahl der Herzcontractionen in 30". Chininapplication. 
Das Herz stark gefüllt mit dunklem Blut; Diastole länger als Systole davernd. Reflexe sehr schwach. In den Versuchen an Fröschen, in welchen von 0,020 bis $0,025 \mathrm{gr}$ Chinin gegeben war, sah man nach $1 \frac{1}{2}-2$ Stunden die Reflexe vollkommen verschwinden. Die Herzcontractionen dauerten jedoch, obwohl sehr schwach und selten, fort.

Versuche an Hunden. Versuch I. Hund ron 5 kgr Körpergewicht, am Operationstisch befestigt. Arteria femoralis mit dem Kymographion verbunden, mit dessen Hülfe und einem Chronometer die Zahl der Herzschläge bestimmt wurde. N. cruralis sin. durchschnitten. Vor Chinineinführung schlug das Herz in $15^{\prime \prime}=26$ Mal. Nach Application von 0,024 gr chin. muriat. unter die Bauchiaut, erhielt man:

Beobachtungszeit nach Chininapplica- Zahl der Herzcontractionen in 15". tion in Ninuten.

Hierauf wurde demselben Hunde $0,104 \mathrm{gr}$ chinin muriat. in wässeriger Lösung in das rectum eingeführt. Die Pulszahl nach Verlauf von 1 Stunde und 14 Minuten seit der Application $=42$ in 15". Endlich nach einer zweiten Beibringung von 0,104 gr chin. mur. in derselben Form in den Mastdarm, war die Pulszahl auf 50 in $15^{\prime \prime}$ nach Verlauf von einer Stunde gestiegen.

Versuch II. Ein $5 \mathrm{kgr}$ schwerer Hund; Tracheotomie zur leichten Einführung der Chininlösung in den Magen mittelst der Scblundsonde. Art. carotis sin. mit dem Kymograph verbunden; N. vagus sin. durchschnitten. Pulszahl in $15^{\prime \prime}$, vor Chinin $=24$. Nach der Einführung vou 0,650 gr Chinin in den Magen.

Beobachtungszeit in Minuten nach Zahl der Herzcontractionen in 56". Chininapplication.

$51 \quad 37$

$74 \quad 42$

$90 \quad 48$

Versuch III. Hund von $6 \mathrm{kgr}$ Körpergewicht. Art. carotis dextrà mit dem Kymographion verbunden; N. vagus dexter durchschnitten. Pulszahl vor Chinin in $15^{\prime \prime}=23$. Nach Injection von $0,120 \mathrm{gr}$ Chinin in Lösung in die rechte Schenkelvene erhielt man:

Beobachtungszeit in Minuten nach . Zahl der Herzcontractionen in 15". Chininapplication.

$30^{4}$ nach einer zweiten Injection von $0,090 \mathrm{gr}$. Chinin in dieselbe Vene war die Pulszahl 44 in $15^{\prime \prime}$. Endlich injicirte man in dieselbe Vene noch $0,120 \mathrm{gr}$ Chinin und die Pulszahl war nach 30 Minuten auf 48 in 15" gestiegen. 
Selbstständige Contraction d. Herzspitze, Veränderungen d. Muskeln etc. 59

Versuche àn Menschen. Ausser den Versuchen an Fröschen und Hunden, machte ich noch zwei Beobachtungen an mir selber behufs Controle der Chininwirkung auf die Herzcontractionen des Menschen. Zu diesem Zweck nahm ich nüchtern in 1 Versuche $0,875 \mathrm{gr}$ und im 2. 1,0 $\mathrm{gr}$ Chinin muriat. in wässeriger Lösung ein. Während des Versuchs verhielt ich mich vollkommen ruhig. Im ersten Versuch erhob sich die Pulszahl

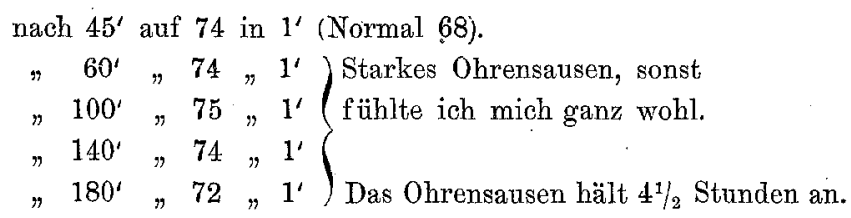

Im zweiten Versuche stieg die Pulszahl nach einer Stunde auf 78 in der Minute (normal 72).

Nach $1 \frac{1}{2}$ Stunden auf 84

$\begin{array}{lllll} & 2 & n & & 84 \\ & 21 / 2 & n & & 78 \\ & 3 & n & & 84\end{array}$

Sieben Stunden andauerndes starkes Ohrensausen. Ich fühlte mich ganz wohl, nur bemerkte ich an den Extremitäten ein schwaches Zittern.

Aus allen diesen. Versuchen geht hervor, dass die Herzcontractionen unter Chinineinfluss beim Frosch grösstentheils sich verlangsamen, dagegen beim Hunde und Menschen sich beschleunigen. Eine Verlangsamung der Herzcontractionen bei letzteren Thieren konnte ich im Gegensatz zu einigen frïheren Beobachtungen nicht wahrnehmen; es ist möglich, dass zur Herbeiführung dieser Erscheinung verhältnissmässig grössere Dosen von Chinin oder ein besonderer Zustand der Thiere und des Menschen nothwendig sind.

Es fragt sich nun, wodurch kann man sich die Beschleunigung oder Verlangsamung der Herzcontractionen nach Chinin erklären?

Die Vermehrung der Herzschläge kann eintreten entweder durch verminderte Thätigkeit des Hemmungsapparates, oder in Folge vermehrter Thätigkeit der motorischen Nervencentra, oder endlich von gradueller Veränderung der Reizbarkeit der Muskelelemente des Herzens.

Nach Levizki's ${ }^{1}$ ) Angaben hat das Chinin keinen Einfluss auf den $\mathrm{N}$. vagus, während $\mathrm{B}$ in $\mathrm{z}^{1}$ ) eine geringe Depression dieses Nerven constatirte, und Jerusalimsky die Wirkung des Chinins auf $N$. vagus der des Atropins gleichsetzt. Während nun die eben

1) Virchow's Archiv. 
angeführten Angaben von Levizky und Binz theilweise gerechtfertigt erscheinen, ist die von Jerusalimsky entschieden irrig. Ueber die Wirkung des Chinins auf den $\mathrm{N}$. vagus kam ich auf Grund einiger Beobachtungen zur folgenden Ueberzeugung.

Der Hemmungsapparat von Fröschen wird nicht besonders von kleinen Dosen Chinin, welche zuweilen Beschleunigung, häufiger aber Verlangsamung der Herzcontractionen herbeiführen, beeinflusst. Schwache Reizung des N. vagus oder des Herzens an der Grenze zwischen den Ventrikeln und den Vorhöfen, ruft, nach vorausgegangener subcutaner Application von kleinen Dosen eines Chininsalzes beim Frosch einen vollständigen Herzstillstand in der Diastole hervor. Mittlere und grosse Gaben des Chininsalzes paralysiren den Hemmungsapparat des Herzens, weil man durch electrische Reizung der erwähnten Abschnitte des herausgenommenen Herzens nicht Stillstand in Diastole, sondern im Gegentheil $\mathrm{Zu}$ nahme der Herzschläge erhält. Von solchen Dosen beobachtet man aber bei den Fröschen nicht Vermehrung, wie gewöhnlich bei Atropin, sondern Verlangsamung der Herzcontractionen.

Folglich existirt nicht die geringste Análogie in den Wirkungen des Chinins und des Atropins auf das Froschberz. Zur Bestätigung: des Gesagten seien hier folgende zwei Versuche angeführt.

Bei einem kleinen Frosche das Herz freipräparirt. Nach einiger Erholung wurden in $30^{\prime \prime}=23$ Herzschläge gezählt. Hierauf subcutane Application von 0,004 gr chin. muriat. in wässeriger Lösung; nach $45^{\prime}$ die Zahl der Herzcontractionen = 19 in $30^{\prime \prime}$. Das herausgeschnittene Herz wurde nun auf eine Messingplatte gelegt, welche einerseits mit einem höehst beweglichen Hebel and andererseits mit den Electroden eines Inductionapparats verbunden war, so dass man jède Veränderung der Herzcontractionen graphisch auf der Trommel vom Kymograph erhielt und das Herz nach Wunsch durch einen Inductionsstrom von bestimmter Stärke reizen konnte ${ }^{1}$ ). Nach Reizung eines solehen Herzens erhielt man seinen vollkommenen Stillstand in der Diastole, wie man es aus der weiter unten folgenden Darstellung ersieht. Beim zweiten Ver-

1) Der zu diesem Zwecke benutzte Apparat im Laboratorium von Prof. Dogiel wird in kurzer Zeit in einer Abhandlung von $A_{\text {ristow beschrieben }}$ werden. 
such war die Zahl der Herzschläge nach Freilegung des Herzens und Erholung desselben von der Operation $=22$ in $30^{\prime \prime}$.

Hierauf führte man unter die Schenkelhaut des Frosches 0,010 gr ehin. mur. in wässeriger Lösung; nach $35^{\prime}$ erhielt man nur 17 Herzschläge in $30^{\prime \prime}$. Nach Reizung des nun herausgeschnittenen Herzens in oben beschriebener Weise stand es nicht in Diastole still, sondern fing sogar schneller an fortzuschlagen.

Bei Hunden wirkt das Chinin entweder gar nicht oder schwach auf den $\mathrm{N}$. vagus ein, auch bei solchen Dosen, welche die Herzschläge bedeutend vermehren. So wurde bei einem $2 \frac{1}{2} \mathrm{kgr}$ schweren jungen, an den Operationstisch befestigten Hund die Art. carotis sin. mit dem Kymograph verbunden und der N. vagus sin. durchschnitten. Die Zahl der Herzschläge $=27$ in $10^{\prime \prime}$; nach Reizung des peripheren Stumpfes vom durchschnittenen Nerv mit einem schwachen Inductionsstrom (1 Element und Inductionsapparat von Gaiff, bei einem Rollenabstand von $1 \mathrm{~cm}$ ) erhielt man nur 10 Schläge in $10^{\prime \prime}$. 30 Minuten nach Injection von $0,120 \mathrm{gr}$ Chin. muriat. in wässeriger Lösung in venam saphenam dextram, stieg die Zahl der Herzschläge auf 40 in $10^{\prime \prime}$; die Zàhl fiel auf 25 bei Reizung nach obiger Art; bei Reizung des peripherischen Stumpfes des durchschnittenen rechten Vagus - 10" lang mit ebenso starkem Inductions-Strom erhielt man aber nur 12 Schläge in $10^{\prime \prime}$. Solche Versuche wurden mit fast gleichem Resultat einige Male wiederholt. Folglich wird die Wirkung des Chinins auf den Vagus des Hundes und wahrscheinlich auch des Menschen eine sehr geringe sein.

Auf Grundlage dessen muss die Vermehrung der Herzcontractionen bei diesen Thieren und beim Menschen nicht vom paretischen Zustand des Hemmungsapparats, sondern von verstärkter Thätigkeit der motorischen Nervencentra des Herzens abhängen; aber auch diese können von grossen Dosen Chinins paralysirt werden, wodurch man auch die Abnahme der Zahl der Herzschläge bei Fröschen, von welchen oben die Rede war, sich erklären kann. Selbstverständlich können an der Verlangsamung der Herzcontractionen auch die Muskelelemente des Herzens Theil nehmen. 
Veränderung der Reizbarkeit der Herz- und Scelettmuskeln.

Viele Beobachter der Chininwirkung auf den thierischen Organismus (Jolyet, Levizki, A. Eulenburg u. A.) nehmen an, dass das Chinin die Herzmuskulatur stark beeinflusst, ihre Thätigkeit nämlich, sich zu contrahiren, vermindert. Dadurch wollte man sogar die Abnahme der Zahl der Herzschläge nach Chinin erklären. Dieser Schluss, wenn auch nicht ganz irrig, ist in dem Falle nur, in welehem die Chiningabe sehr gross genommen wird, gerechtfertigt; in anderen Fällen ist die Erregbarkeit des Herzmuskels wie des "Scelettmuskels nicht nur nicht kleiner, sondern sogar erhöht. Ueber die Wahrheit des soeben Gesagten überzeugte ich mich auf folgende Art.

Das unterste Drittel des Froschherzens eńthält, wie bekannt, keine Nervenzellen, wie die Scheidewand der Vorhöfe und Grenze zwischen den Vorhöfen und den Ventrikeln. Dieser Theil des Herzens contrahirt sich nach Abtrennung nicht mehr rhythmisch, wie die anderen Herztheile, obwohl er noch auf mechanischen oder electrischen Reiz reagirt. Im Gegensatz zu dem fährt das untere Drittel des Herzens von mit Chinin vergifteten Fröschen fort, sich ziemlich lange zu contrahiren. Ich will etliche von der grossen Zahl in dieser Beziehung angestellten Versuche hier vorführen.

Versuch I. Das Herz eines kleinen Frosches frei gelegt und dem Thier Erholung gegönnt. Zahl der Herzschläge 24 in $30^{\prime \prime}$. Unter die Schenkelhaut $0,002 \mathrm{gr}$ chin. sulf. in wässeriger Lösung gefïhrt; nach 12 Minuten

$\left.\begin{array}{l}\text { Zahl der Herzschlïge . . . . . 22 } \\ \text { Nach } 50 \text { Min. Zahl der Herzschläge } 23\end{array}\right\}$ in $30^{\prime \prime}$.

Die Herzcontractionen kräftig. Reflectorische Erregbarkeit etwas erhöht. Mit einer kleinen Scheere das unterste Drittel des Herzventrikels (Herzspitze?) abgeschnitten. In 4" machte es 7 Zusammenziehungen; darauf trat nach jedem leichten mechanischen Reiz eine starke Contraction auf.

Versuch II. Kleiner Frosch. Das Herz freigelegt und Erholung. Zahl der Hersschläge $=25$ in $30^{\prime \prime}$. Nach Application von 0,004 gr chin. muriat. wässeriger Lösung unter die Rückenhaut, war die Zahl der Herzcontractionen

$$
\left.\begin{array}{l}
\text { nach } 15^{\prime}=28 \\
\text { nach } 45^{\prime}=19
\end{array}\right\} \text { in } 30^{\prime \prime} .
$$

Hierauf wurde die Herzspitze abgeschnitten, welche sogleich anfing, sich 
zu contrahiren. In den ersten $30^{\prime \prime}$ fielen ihre Contractionen bald mit Systole, bald mit Diastole des übrigen Herzens zusammen, endlich wurden die Contractionen allmählig schwächer, bis sie völlig aufhörten, was $2 \frac{1}{2}$ Minuten nach oben ausgeführter Operation eintrat. Einige Minuten am Anfange der Ruhe reagirte die Herzspitze auf jeden leichten mechanischen Reiz mit einmaliger Contraction.

Versuch III. Einem Frosch mittlerer Grösse injicirte man unter die Rückenhaut 0,014 chin. mur. in wässeriger Lösung. Vor der Chininapplication waren 20 Herzschläge in $30^{\prime \prime} ; 40^{\prime}$ nach der Injection $=15$ in $30^{\prime \prime}$. Reflexe schwächer als im normalen Zustande. Die abgetrennte Herzspitze zog sich im Verlaufe von 7 Minuten noch zusammen, wornach die Contractionen, allmählich schwächer werdend, aufhörten.

Nach dem Vorschlag und der Methode Bernstein's änderte ich die Ansführung meiner Versuche in der Weise ab, dass ich statt die Herzspitze abzuschneiden, ihren peripherischen Zusammenhang mit anderen Herztheilen durch starken Druck mit einer Pincette oder auf irgend eine andere Art aufhob.

War der Frosch vordem nicht mit Chinin vergiftet, so sah man die Herzspitze sich nicht mehr rhythmisch zusammenziehen, obwohl es mit demselben Blut wie die anderen Herztheile versorgt wird. Umgekehrt contrahirt sich bei Chininvergiftung die Herzspitze, und die Contractionen halten lange an, wie man aus folgendem Versuch ersieht.

Versuch IV. Einem mittelgrossen Frosch injicirte man 0,016 gr chin. mur. in wässeriger Lösung unter die Rückenhaut. $50^{\prime}$ darnach wurde das Herz freigelegt, es contrahirte sich $12 \mathrm{Mal}$ in $30^{\prime \prime}$. Mittelst einer Pincette, deren Spitzen vollkommen glatt und rund waren, wurde nun ein starker Druck zwischen dem mittleren und untersten Drittel des Herzventrikels ausgefïhrt. Nach Aufhören des physiologischen Zusammenhangs der Herzspitze von den übrigen Herztheilen, dauerte die selbstständige Contraction auch im untersten Drittel des Herzventrikels fort. Die Contraction der Herzspitze entsprach mehr der Diastole als der Systole der übrigen Herztheile. In der ersten Minute contrahirte sie sich ebenso oft wie die Vorhöfe und der obere Theil des Ventrikels, worauf aber. eine Verlangsamung um 2 bis 3 Male auftrat. Nach 5 Minuten wurde die sich contrahirende Herzspitze abgeschnitten, worauf sie sich noch 4 Minuten weiter zusammenzog. Machte man nun, nach dem sie aufgehört hatte, sich zusammenzuziehen, einen Schnitt parallel dem, der sie vom Herzen trennte, so fing die nun kleinere Herzspitze wieder an, sich zu contrahiren, und dieses dauerte $1 \frac{1}{2}$ Minuten an.

Bei einem andern mit 0,014 gr chin. mur. vergifteten Frosch dauerten die Contractionen der auf obige Art isolirten Herzspitze 20 Min. an. Das 
Angeführte über die Herzspitze der Frösche nach Chininapplication kann man auch am Herz der Hunde und Kaninchen demonstriren. So wurde einem jungen Hund von 21/2 Kilo Körpergewicht, 0,120 gr chin. mur. in wässeriger Lösung in venam saph. sinist. geführt. Nach 32 Minuten zählte man 240 Herzschläge in der Minute (normal 162). Die Brusthöhle wurde geöffnet und die Spitze des schlagenden Herzens abgetrennt. Diese machte 5 Contractionen, aber im Vexlauf von 12 Minuten war der kleinste mechanische oder schwächste electrische Reiz genügend, um eine einmalige, energische Contraction auszulösen. Das ibrige Herz contrahirte sich nach der Operation noch $7 \mathrm{Min}$. lang. Alle diese Beobachtungen an der abgetrennten Herzspitze der Frösche, wie Warmblüter (Hund, Kaninchen), führen zu dem Schluss, dass die Erregbarkeit der Herzmuskelfasern durch Chinin stark erhöht ist. Da aber die Herzspitze im gegebenen Falle selbstständig sich zusammenzieht, dabei keine Nervenzellen enthält, so fragt es sich, wodurch die Contractionen zu erklären sind? Indem ich den Bewegungen der Herzspitze sorgfältig folgte, sah ich, dass die Contractionen sofort aufhörten, wenn sich die Schnittflächen mit Blutcoagula bedeckten; wurden die Coagula entfernt oder durch einen neuen Schnitt die Herzspitze wieder mit Chinin haltendem Blut in Berïhrung gebracht, so fing sie sich wieder an zusammenzuziehen. Weiter contrahirte sich die durch Druck von den übrigen Herztheilen isolirte Herzspitze längere Zeit (wobei ihre Contractionen mit der Diastole des ibrigen Herzen zusammenfielen), als die abgeschnittene Herzspitze. Alles das spricht dafür, dass das Stimulans für die Herzspitze das mit Chinin vergiftete Blut ist. Indem es von Zeit zu Zeit die Muskelelemente reizt, ruft es so lange Contractionen hervor, bis die Fähigkeit, solche auszuführen, nicht völlig erloschen ist. Bei den nicht abgeschnittenen Herzspitzen nun bilden sich keine Blutgerinnsel, welche die Contractionen verhindern könnten, folglich contrahiren sie sich anch länger, als die abgeschnittene Herzspitze.

Diese Erklärung über die nächste Ursache der Herzspitzencontractionen wird durch den Umstand bekräftigt, dass auch die Erregbarkeit der Scelettmuskeln in gewisser Periode der Chininwirkung erhöht ist. Und wahrlich, wenn man das Maximum der Contractionskraft der m. gastrocnemii des Frosches an einem Beine durch Reizung mit einem Inductionsstrome bestimmter Stärke, mit dem Maximum der Kraft des entsprechenden Muskels des anderen Beines desselben Frosehes, nach Vergiftung mit Chinin vergleicht, 
so ergibt es sich, wie die später folgende Darstellung zeigt, dass das Maximum der Contractionskraft des letzteren Muskels fast zweimal so gross ist, was aus folgender Figur zu ersehen ist.

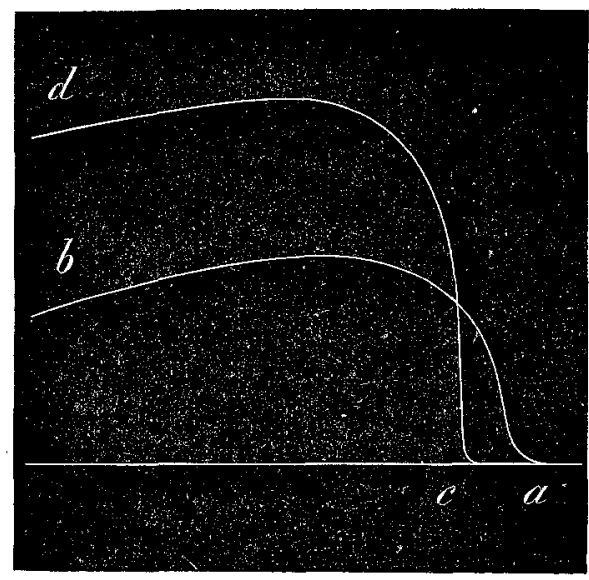

\author{
von a bis $b$ Maxi- \\ mum der Con- \\ traction des $\mathrm{M}$. \\ gastrocnemins \\ vor der Vergif- \\ tung. \\ von $\mathrm{c}$ bis d Maxi- \\ mum der Con- \\ traction des M. \\ gastrocnemius \\ nach der Ver- \\ giftung.
}

Es muss aber bemerkt werden, dass die Erregbarkeit des M. gastrocnemius bei weiterer Einwirkung des Chinins fällt, und zwar zu einer Zeit, in welcher die abgeschnittene Herzspitze noch automatisch fortschlägt. Dieses zwingt seinerseits zum Schluss, dass die Erregbarkeit der Muskelelemente des Herzens länger andauert, als die der Skelettnuskeln. Bei starken toxischen Gaben des Chinins verändert sich auch bei Fröschen das Muskelgewebe des Herzens, so dass die abgetrennte Herzspitze nicht nur nicht automatisch sich zusammenzieht, sondern sogar auf mechanischen oder electrischen Reiz schwach oder gar nicht reagirt.

Die stark erhöhte Erregbarkeit der Herzmuskelfasern in gewisser Periode von Chininwirkung, bleibt gewiss nicht ohne Einfluss bei den Veränderungen der Herzschläge durch dieses Mittel. Da aber die erhöhte Erregbarkeit auch zu einer Zeit, in welcher bei Fröschen schon Verlangsamung der Herzschläge eingetreten ist, erscheint, so kann man mit den Schlussfolgerungen einiger Beobachter, welche die Verlangsamung durch verringerte Contractionsfähigkeit der Herzmuskeln erklären, nicht einverstanden sein. Rechnet man hierzu noch die Thatsache, dass bei der starken Verlangsamung der Herzschläge beim Frosch durch Chinin ein E. Pflüger, Archiv f. Physiologie. Bd. XIX. 
66 Schtschepotjew: Selbstständige Contraction der Herzspitze etc.

paralytischer Zustand des Hemmungsapparats besteht, während die abgetrennte Herzspitze noch fähig ist, sich automatisch zu contrahiren, so muss man unumgänglich annehmen, dass bei der Verlangsamung der Herzschläge beim Frosch und bei der Beschleunigung des Pulses beim Hund und. Mensch, hauptsächlich die motorichen Centra des Herzens theilnehmen, indem ihre Thätigkeit bald erhöht, bald geschwächt wird. 